\title{
Fine-grained Emotion Role Detection Based on Retweet Information
}

\author{
ZHIWEN YU, Northwestern Polytechnical University, P.R.China \\ FEI YI, Northwestern Polytechnical University, P.R.China \\ CHAO MA, Northwestern Polytechnical University, P.R.China \\ ZHU WANG, Northwestern Polytechnical University, P.R.China \\ BIN GUO, Northwestern Polytechnical University, P.R.China \\ LIMING CHEN, De Montfort University, UK
}

User behaviors in online social networks convey not only literal information but also one's emotion attitudes towards the information. To compute this attitude, we define the concept of emotion role as the concentrated reflection of a user's online emotional characteristics. Emotion role detection aims to better understand the structure and sentiments of online social networks and support further analysis, e.g., revealing public opinions, providing personalized recommendations, and detecting influential users. In this paper, we first introduce the definition of a fine-grained emotion role, which consists of two dimensions: emotion orientation (i.e., positive, negative, and neutral) and emotion influence (i.e., leader and follower). We then propose a Multi-dimensional Emotion Role Mining model, named as MERM, to determine a user's emotion role in online social networks. Specifically, we tend to identify emotion roles by combining a set of features that reflect a user's online emotional status, including degree of emotional characteristics, accumulated emotion preference, structural factor, temporal factor and emotion change factor. Experiment results on a real-life micro-blog reposting dataset show that the classification accuracy of the proposed model can achieve up to $90.1 \%$.

CCS Concepts: • Information systems $\rightarrow$ Social networks; Data mining; Web mining;

Additional Key Words and Phrases: Online Social Networks, Emotion Role, Repost Information

ACM Reference Format:

Zhiwen Yu, Fei Yi, Chao Ma, Zhu Wang, Bin Guo, and Liming Chen. 2018. Fine-grained Emotion Role Detection Based on Retweet Information. ACM Trans. Internet Technol. 9, 4, Article 39 (March 2018), 23 pages. https://doi.org/0000001.0000001

\section{INTRODUCTION}

Over the past few years, online social networking and associated applications have become prevalent and widely adopted, many intelligent systems are developed [6, 40, 43]. Users can freely and conveniently express their opinions through these emerging social networking services such as Twitter and Weibo. These social behaviors not only convey literal information but also user's emotional attitudes towards the information. In particular, user's emotional characteristic is one of

Authors' addresses: Zhiwen Yu, Northwestern Polytechnical University, 127 West Youyi Rd, Xi'an, Shaanxi, 710072, P.R.China, zhiwenyu@nwpu.edu.cn; Fei Yi, Northwestern Polytechnical University, Xi'an, P.R.China, yifeinwpu@gmail.com; Chao Ma, Northwestern Polytechnical University, Xi'an, P.R.China, chaoma@mail.nwpu.edu.cn; Zhu Wang, Northwestern Polytechnical University, Xi'an, P.R.China, wangzhu@nwpu.edu.cn; Bin Guo, Northwestern Polytechnical University, Xi'an, P.R.China, guob@nwpu.edu.cn; Liming Chen, De Montfort University, School of Computer Science, Leicester, UK, liming.chen@dmu.ac.uk.

Permission to make digital or hard copies of all or part of this work for personal or classroom use is granted without fee provided that copies are not made or distributed for profit or commercial advantage and that copies bear this notice and the full citation on the first page. Copyrights for components of this work owned by others than the author(s) must be honored. Abstracting with credit is permitted. To copy otherwise, or republish, to post on servers or to redistribute to lists, requires prior specific permission and/or a fee. Request permissions from permissions@acm.org.

(C) 2018 Copyright held by the owner/author(s). Publication rights licensed to the Association for Computing Machinery. 1533-5399/2018/3-ART39 \$15.00

https://doi.org/0000001.0000001 
the most important factors to help us understand human behavior patterns, which correlates to a user's social behaviors, living habits and other personalities. As a matter of fact, people usually have different emotional characteristics and preferences when they interact with online information. In order to systematically study a user's distinct emotion preferences during information propagation in online social network, we propose the concept of emotion role, which consists of emotion orientation and emotion influence, to denote the concentrated reflection of a user's online emotional characteristics. Specifically, emotion orientation represents a user's own tendency in delivering emotions, e.g., some people prefer to share and publish positive posts while others prefer negative ones. Emotion influence mainly determines to what extent a user could make impact on the propagation of online emotions, which is another significant feature to reflect one's emotional characteristics.

Revealing the emotion role and its impact is crucial for better understanding online social networks and providing critical insights, which can be used to facilitate public opinion detection and personalized information recommendation. For example, [44] exploited the effectiveness of sentiment homophily for link prediction in social network, and [37] took advantages from users' sentiment for fine-grained social media search. However, most related studies focus on how to measure personal-level and short-term emotions, relatively fewer have considered people's longlasting emotion characteristics, such as emotion orientation and emotion influence [30, 32, 38] which will be mainly discussed in our work. And taking these two factors can be of great value for many applications. For example, online product promotion and targeted information diffusion are two meaningful areas within which emotion role detection plays a critically role. When a company releases a product online, the positive or negative comments of the customers on the product's advertisement will significantly impact the image and/or sale of the product. As such, diffusing advertisements by influential users with positive emotional comments in online social network is a promising approach for product promotion. In order to achieve this, it is crucial to determine which online user is influential, and what emotional preference (positive or negative) he/she possesses. In other words, to detect the emotion role of online users is the basis for efficient online product promotion or information diffusion. In addition to marketing, emotion detection can be used for many other situations which have been studied before $[8,12,38]$. This motivates us to extend those traditional models to improve the performance of emotion role detection.

Usually, emotion role detection involves in a few basic methods, which includes emotion recognition, user profiling and social network analysis. In specific, emotion recognition is the foundation for user's emotion role detection, it provides researchers with the basic knowledge of a user's static emotion status and that can be applied in revealing user's emotional characteristic. Besides, social network analysis can also benefit the emotion role detection process, which could help us understand users' historical interactions and their behavior influence. However, despite these existing methods for emotion role detection, there are still a number of challenges which includes 1) what features could be used to describe a user's emotion characteristics; and 2) how to quantify extracted features and fuse them together.

To address the emotion role detection issue, in this paper, we formulate the problem based on two emotional perspectives. The first one is referred to emotion orientation, which determines a user's online behaviors and indicates what kind of emotion a user tends to convey in online social network. We use both degree of emotional characteristics and one's accumulated emotion preference to represent a user's emotion orientation. The second one is emotion influence. In particular, one's behavior in online social network has specific impacts on their followers which may result in different diffusion mode. Different from traditional social influence that only considers the scale of propagation, emotion influence takes the ability of leading emotions into account. Thus, we define users with high emotion influence will not only have topological impact but also play an 
important role in emotion propagation. Above two perspectives build the basis of emotion role in terms of personal-level and public-level respectively, based on which we further extract a number of features and propose a Multi-dimensional Emotion Role Mining model (MERM), to identify the emotion roles of the users of online social network. The main contributions of our work can be summarized as follows:

- We define a fine-grained two-dimension emotion role, by combining both emotion orientation and emotion influence, which is, to the best of our knowledge, the first attempts in the domain of online social network analysis.

- We design a few new features and develop a decision tree-based MERM model to detect the emotion role of online social network users.

- We conduct a set of experiments on real data set, which consists of more than 2.6 million reposts. Experimental results show that the classification accuracy of the proposed model can reach $90.1 \%$.

The remaining of the paper is organized as follows. Section 2 introduces related work. Section 3 describes the concept and definition of emotion role. Section 4 presents the problem statement. Section 5 formulates the features for emotion role detection and further elaborates the proposed MERM model for feature fusion. Section 6 discusses experimental evaluation followed by conclusions and future research directions in Section 7.

\section{RELATED WORK}

\subsection{Emotion Recognition and Propagation}

In early works, [24] conducted a survey on social network analysis, and they took user's semantics as one significant factor for understanding social behavior. Generally speaking, as for emotion/semantic recognition, most of the state-of-the-art methods are implemented by either lexicon-based or learning-based approaches. Lexicon-based approaches typically use existing emotion lexicons of words and phrases, each of which is related to the emotion polarity or strength. [17] constructed a microblog-oriented emotion lexicon using the Semantic Orientation from Pointwised Mutual information and proposed an analysis algorithm to calculate the emotion score. In [4], authors analyzed the emotion of post messages by combining opinion strength and polarity indicators based on existing lexical resources, which significantly improved the accuracy of emotion classification. For the learning-based approaches, [22, 23] have conducted machine-learning based sentiment analysis in early years, and many learning methods have been applied such as Naïve Bayes and Support Vector Machine (SVM) [25]. While most existing sentiment classification algorithms build sentiment classifiers based on the segmentation results from bag-of-words or separators, [31] developed a joint framework for sentence-level sentiment classification which is able to generate more useful segmentations and predict sentence-level polarity. [20] developed a system named pSenti, which integrated both lexicon-based and learning-based approaches, and achieved significantly higher accuracy in polarity classification. In addition, [12] investigated emotional signals to help with sentiment analysis. [46] performed emotion tagging for online comments based on cross-domain resources and [13] exploited social relations for sentiment analysis, which gave us a hint to mining user's emotional behaviors. There are also a few studies that focus on emotion propagation. [34] proposed a trust-based multi-ego social network to investigate and understand dynamic emotion diffusion. [8] characterized the factors influencing the diffusion of emotion in Facebook by using the independent-Cascade diffusion model. 


\subsection{Personal-level Emotion Detection}

This category of related work is about how to measure each individual's static emotion status and its influence. In [38], authors extracted individuals' emotions by jointly modeling the images posted by an online user and the comments added by his friends, which improved the performance in inferring user emotions. [32] conducted a quantitative study on individual emotional states and proposed a method referred to as MoodCast for modeling and predicting emotions. The authors formalized the problem as a factor graph model and defined three types of factors, namely attribute correlation, temporal correlation and social correlation, to capture different types of information in the social network. [30] utilized social relationships to improve user-level sentiment analysis, and constructed models based on the follower/followee network which is formed by the referring actions using "@" mentions. The result has proved that statistically significant sentiment-classification improvements can be achieved by incorporating social-network information. In [15], a framework was proposed to classify emotions and reveal emotion influence patterns. In general, these studies provide us with insights into an individual's online emotion behaviors and relevant factors. This offers us a hint to further analyze user's emotional characteristics.

\subsection{Role Detection in Online Social Network}

This category focuses on approaches to mining social roles according to different role definitions. [33] proposed to assign Twitter users to different levels of status based on their language use, and [14] presented method for detecting organizational hierarchy that aimed to determine the degree to which team members coordinate with each other. Besides, [26] proposed a model called the Longitudinal User Centered Influence (LUCI). The model can discover leaders and followers in online social network and further divide each category into two subcategories. It took the user interaction information as input and achieved high classification accuracy. In [1], the authors presented SSRM framework to analyze social network and classified each individual into four different categories based on their structural role in a community. The closeness centrality and two extensions of the betweenness centrality have been used to extract different social roles. This method has also been used in [3]. [45] introduced Interest-field Based Measures to identify opinion leaders. [47] investigated the social roles and statuses that people act in online social networks from the perspective of network structures. They systematically studied the correlation between network characteristics and social situations of users, and proposed a model called SRS to infer social roles such as advisor, content contributor, etc. In [9], authors proposed several theories about social influence network and explored the opinion change issue which inspires us to further analyze the online social network. However, neither of these definitions or classification approaches had considered a user's long-lasting emotional status. In this paper, we would pay more attention to a user's emotional characteristics and propose a new definition of emotion roles.

\section{EMOTION ROLE DEFINITION}

The emotion role is the concentrated reflection of a user's online emotional characteristics. As mentioned above, we focus on two dimensions of emotion. 1) The first one is emotion orientation, namely the types of emotion a user prefers to express. This dimension includes three different values, i.e., positive, neutral and negative respectively. 2) The second dimension is emotion influence, which indicates one's ability to lead public emotion and is classified into two categories, namely emotion leader and emotion follower.

To classify a user's emotion orientation, we consider two factors, namely 1) degree of emotional characteristics and 2) accumulated emotion preference. As we already know, users may have different emotion attitudes towards the same topic, and prior studies have paid much attention to 
mining such attitudes from personal-level (i.e., micro-level) data [32, 38]. However, the emotion distribution of the public (i.e., macro-level) was barely considered. It remains an open issue to measure the relationships between micro-level behaviors and macro-level behaviors, and their impact on online social network. Emotion preference provides an intuitive insight into a user's historical behaviors and has been explored in many studies [16, 21], which made an important contribution to refine the user's emotion orientation.

For the detection of emotion influence, we take into account three factors: namely 1) structural factor - a traditional perspective to measure influence, 2) temporal factor - representing activeness and temporal influence, and 3) emotion change factor - denoting the possible emotion change caused by the user during propagation of information. The last one plays an important role in identifying the key node that could lead public emotions in the process of information propagation, but it received little attention in existing researches. Based on the above analysis, and by combining the three types of emotion orientation with two kinds of emotion influence category, we define six distinct types of emotion roles as follows:

- Positive Leader. The online social network users whose behaviors are mostly inclined to be positive and could make a strong impact on emotion propagation.

- Positive Follower. The online social network users whose behaviors are mostly inclined to be positive but are barely followed. Compared with positive leaders, positive followers could not lead any meaningful propagation of emotions.

- Negative Leader. The online social network users whose behaviors are mostly inclined to be negative and could lead to a wide range propagation of emotions.

- Negative Follower. The online social network users whose behaviors are mostly inclined to be negative, but their emotions could hardly spread out and affect others.

- Neutral Leader. The online social network users whose behaviors are mostly inclined to be neutral, which includes the following two meanings: The first one is that online behaviors of neutral leaders do not always have polarity tendency, i.e., their behaviors are mostly inclined to be objective and rational. The second one is that online behaviors of neutral leaders do not have a specific mode, which might randomly be positive, negative or neutral. Meanwhile, their behaviors could still make an impact on others and result in emotion changes.

- Neutral Follower. The online social network users who neither have obvious emotion orientation or any meaningful influence to others. The majority of online users belong to this type.

\section{PROBLEM STATEMENT}

In online social networks, such as Twitter ${ }^{1}$ and Weibo ${ }^{2}$, users could interact with others in many ways, e.g., making comments, clicking likes, and retweeting/reposting other's messages. Among them the retweet/repost behavior can be used to track the propagation of information. This kind of information propagation naturally forms a tree structure within which users correspond to nodes and retweeting/reposting behaviors correspond to edges [42]. As illustrated in Figure 1, we extract the repost structure in Weibo using the identifier "//@”. In this way, the propagation structure of a micro-blog message can be represented as a tree $T(U, E)$, where $U$ denotes the set of users and $E$ represents the set of repost edges. As a user may get involved in several different propagation trees, there might be a list of trees for each user. In order to formally define the emotion role identification problem, we first introduce the relevant notations below.

\footnotetext{
$\overline{{ }^{1} \text { www.twitter.com }}$

${ }^{2}$ www.weibo.com
} 


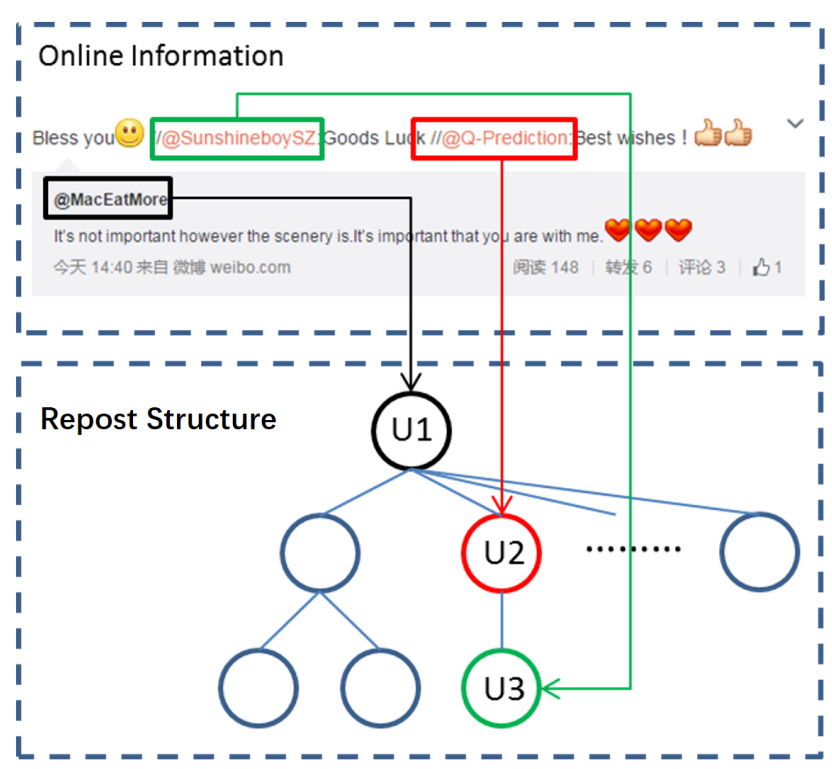

Fig. 1. A structural example of a repost in Weibo.

Let $T_{i}$ denote a repost tree, $u_{i}$ denote an online social network user, $T_{i} \in T, u_{i} \in U$, and $W_{u}$ denote all the posts that a specific user $u$ has ever reposted. We represent the emotion orientation of user $u$ as $E O_{u}$, which consists of the following two parts. On the one hand, the degree of emotional characteristics of user $u$ for post $w$ is represented as $E R_{u}(w)$. On the other hand, the user's accumulated emotion preference is extracted from his/her historical behaviors. We use $H P_{u}(e)$ to represent the historical preference of user $u$ for emotion $e$. We denote the emotion influence of user $u$ as $E I_{u}$, which can be defined based on the following three key features. $T F_{u}(w)$ is the temporal factor of user $u$ for post $w . S F_{u}(w)$ is the structural factor which represents the topological structure of subtrees. $E F_{u}(w)$ denotes the emotion change caused by user $u$.

Therefore, the emotion role identification problem can be described as: given a user $u$ and a set of trees (i.e., $T$ ) related to the user's repost history, we aim to identify which role the target user $u$ belongs to. The key tasks are outlined as follows:

- How to quantitatively evaluate the aforementioned features, including $E R_{u}(w), H P_{u}(e)$, $T F_{u}(w), S F_{u}(w)$ and $E F_{u}(w)$ ?

- How to fuse these factors together to determine the user's emotion role?

\section{EMOTION ROLE DETECTION}

In this section, we first introduce the preliminaries of emotion recognition from a personal-level perspective, and then elaborate the features used for identifying emotion roles based on emotion orientation and emotion influence. Finally, we present a feature fusion model and its corresponding methods.

\subsection{Preliminaries}

Emotion recognition [2] focuses on the identification of personal-level emotion from textual contents, images or any other resources from online social networks. As all concepts of emotion roles are based on emotion recognition of a user's online behaviors, emotion recognition is critical 


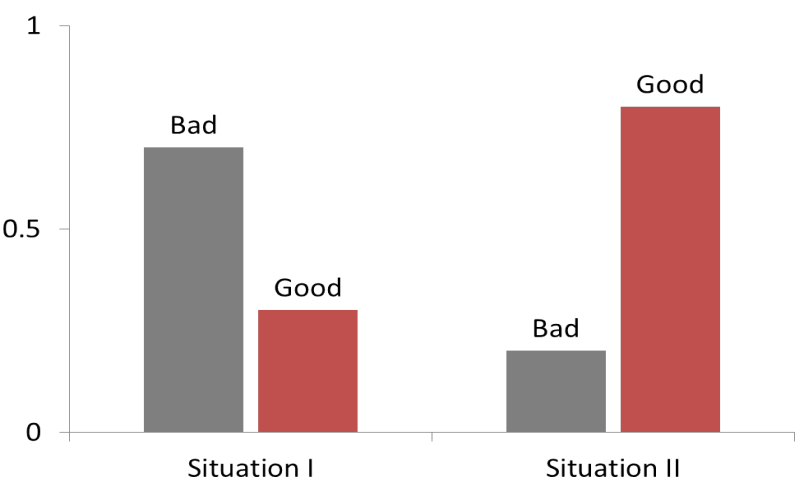

Fig. 2. Illustration of different distribution of emotion.

to thoroughly understand a user's emotion role. Nowadays, many emotion classification tools have been developed based on either lexicon or machine learning methods, which is also one of our previous works [36, 41]. Based on the characteristics of online social networks, [36] paid attention to the characteristics of innovative words, emotion elements, and hierarchical structure of Chinese micro-blog. Based on three wording standards and point-wise metrics, a Rule Set Model (RSM) for analyzing sentiment features of different linguistic components and corresponding methodology for calculating sentiment on multi-granularity were developed. And the method has been proved more effective by giving $10.2 \%$ and $1.5 \%$ higher average accuracy than two existing methods for Chinese micro-blog sentiment analysis, namely $\mathrm{CBoO}$ [35] and OVDB [7]. In this paper, based on textual information, we use RSM to classify personal-level emotion into three categories: $\mathrm{P}$ (positive), $\mathrm{O}$ (neutral), and $\mathrm{N}$ (negative). We use $M$ to denote the candidate emotion set, where $M \in\{P, O, N\}$, and use $E_{u}(w) \in M$ to denote the emotion attitude of user $u$ towards repost $w$.

\subsection{Emotion Feature Extraction}

\subsubsection{Emotion Orientation.}

In online social networks, some people prefer to express positive emotions, and would like to share wonderful experiences or repost encouraging stories. Meanwhile, there is also another type of people who prefers to deliver negative emotions such as publishing rude remarks or expressing dissatisfaction. Emotion orientation reflects a user's online emotion characteristic which is related to the user's living environment, educational background, economic situation, etc. As we know, topic preference could reflect an individual's unique characteristic because different people are usually attracted by different topics, e.g., politics, entertainment news, literature. However, emotion orientation is derived from a completely different perspective, e.g., two users who have totally opposite topic preference may be consistent with each other when it comes to emotion orientation. In other words, emotion orientation, to a certain degree, is independent of topics [19]. For instance, assuming that one user is interested in politics and makes a lot of negative comments on social events (e.g., government policy and international situation), and another user is a sport fan and his online behaviors are always critical and radical. Obviously, they are two completely different kind of people based on topic preference. Nevertheless, in terms of emotion orientation, they can be categorized as the same kind of people, i.e., the negative ones. To quantitatively measure emotion orientation, we propose the following two features. 
Degree of emotional characteristic. By applying the RSM method in previous work [36], we can classify user's posts into three categories of emotion, i.e., positive, negative, and objective. However, these discrete labels are insufficient to measure the fine-grained emotional characteristics, in other word, it is much more effective to understand an emotion role if we could quantify the degree of emotional characteristics for online users. Without loss of generality, online users usually possess different emotions towards the same event, as shown in Figure 2, which shows two situations of emotion distributions. If a user dares to express a different emotion feeling from the others', we can deduce that such a distinct emotion is much more unique than that he/she expresses a similar emotion as the others. For instance, a positive emotion in a situation that other users all expressed negative feeling, is obviously more unique than that in the case all others showed similar positive feelings. Based on this observation and inspired by TF-IDF ${ }^{3}$ method, we attempt to measure the difference between a user's micro emotion feeling and the overall macro emotion distribution to identify the uniqueness of a user's emotional characteristics. Specifically, we extract individual and crowd emotions from the repost structure of a given repost tree, and apply the following equations to quantify the degree of a user's emotional characteristics.

$$
E R_{u}(w)=\left\{\begin{array}{cll}
0.5+\frac{N(w)-P(w)-|N(w)-O(w)|}{2 S(w)} & \text { if } & E_{u}(w)=P, \\
\frac{P(w)-N(w)}{2 S(w)} & \text { if } & E_{u}(w)=O, \\
-0.5+\frac{N(w)-P(w)-|P(w)-O(w)|}{2 S(w)} & \text { if } & E_{u}(w)=N .
\end{array}\right.
$$

where $E R_{u}(w)$ is the degree of emotional characteristics, and $E_{u}(w)$ denote the emotion attitude of user $u$ towards repost $w$, which is a discrete value that can be positive $(\mathrm{P})$, negative $(\mathrm{N})$, or objective (O). $N(w)$ represents the number of users whose emotion towards $w$ is negative; $P(w)$ denotes the number of users whose emotion is positive; $O(w)$ is the number of users whose emotion is neutral; and $S(w)$ represents the total propagation size of this post, i.e., its total reposts number. In this equation, we leverage the difference between $N(w)$ and $P(w)$ to describe the position of a user in the macro emotion distribution and revise it using $O(w)$. To set the value of neutral relations to zero and the value of $E R_{u}(w)$ ranges from -1 to 1 , we set 0.5 as base point of positive relations and -0.5 as negative point.

We use the following example to explain the mechanism of our proposed method for calculating $E R_{u}(w)$. Suppose that 30 users have reposted the same post, among them 20, 7 and 3 users express positive, objective, and negative feelings respectively. According to equation 1, the degree of positive emotional characteristic for the users expressing positive emotion is $0.5+(3-20-|3-7|) / 60=0.15$. Similarly, the corresponding degrees of negative and objective emotional characteristics for users expressing negative or objective emotion are -1 or 0.28 respectively. All these values: 0.15 (positive), 0.28 (objective), and -1 (negative) indicate the degree of the corresponding emotion feelings. As can be seen, since there are 20 users expressing positive emotion, the positive emotion expressed by the user will not be significant comparing to a negative or objective feeling ( 0.15 is smaller compared to -1 and 0.28 in their absolute values) expressed by users. Thus, by applying such equation, we could deduce the degree of userâĂŹs emotional characteristics.

User's accumulated emotion preference. Based on the analysis of users' history behavior, we noticed that although the sequential records of a user's emotion preference remains stable, its temporal fluctuations and diversity contextual information makes it difficult to capture a user's comprehensive emotion preference. Intuitively, we could simply average the value of one's historical emotion preference or apply the last emotion preference as the default user's emotion preference at present moment. However, these methods could lose temporal and contextual information

${ }^{3}$ https://en.wikipedia.org/wiki/Tf\%E2\%80\%93idf\#cite_note-1 


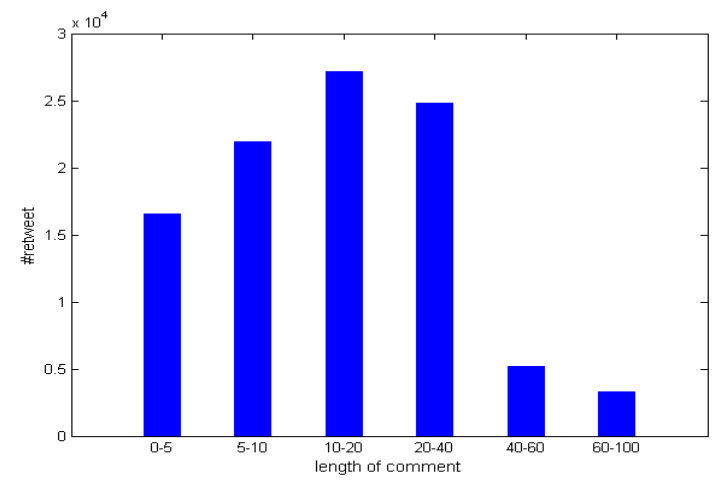

Fig. 3. Distribution of comment length.

that exist in user's history behavior. Therefore, the following factors are taken into account. We first look into the influence of temporal factor, in which we introduce time decay factor when averaging all the historical emotion preferences. And one previous work [28] has proved that recent emotions (moods) are more effective to infer user's current emotion than past emotions. The second observation, as discussed in [27,29], is that the constitution of online comments could have influence on individual's online behavior, including reviewing and posting comments. Specifically, these works propose concepts like "thoughtfulness of existing comments" and "richness of the content" to measure the influence that existing comments could have on individual's behavior. As the conveyance of such concepts is related to comment length to some extent, we take comment length into consideration in our work in modeling user's reposting behavior. Figure 3 illustrates the statistical analysis of the comment length based on our data set, which shows different reposting behavior under different comment length. By combining the comment length and the time decay factor, we can quantify a user's accumulated emotion preference as follows:

$$
H P_{u}(e)=\sum_{E_{u}(w)=e, w \in W_{u}} \log \left(C_{u}(w)+2\right) \exp \left\{-\theta\left(t_{0}-t_{w}\right)\right\}
$$

where $t_{0}$ is the latest time when user $u$ reposted or published a message; $\exp \left\{-\theta\left(t_{0}-t_{w}\right)\right\}$ represents a time-decay factor; and $\theta$ is a attenuation parameter which describes the time-decay trend and is set as 0.2 according to our experiments on the separated training set of our data. $C_{u}(w)$ is the user's comment length of post $w$. We used logarithmic function to eliminate the effect of large orders of magnitude of comment length. Since $C_{u}(w)$ is not lesser than 0 and the base of our logarithmic function is 2, we extend the value of $C_{u}(w)$ with 2 in addition in the logarithm to keep this function meaningful, in other word, to make it larger than 1 in all situations.

\subsubsection{Emotion Influence.}

Emotion influence is a measurement of users' ability to propagate online emotion. We consider this feature from three different perspectives. The first one is structural influence, which is related to the scale of information propagation caused by the user. It is similar to some existing studies, most of which summarized the problem as how to find leaders and followers in online social networks. The second one is temporal factor, which is related to the life cycle of a post. The last one is emotion change after a user's participation. During the propagation of a piece information, the emotion opinion towards it may change with the effect of surrounding context. Specifically, the last factor plays a key role in measuring a user's ability to impact public emotions, which distinguishes the 


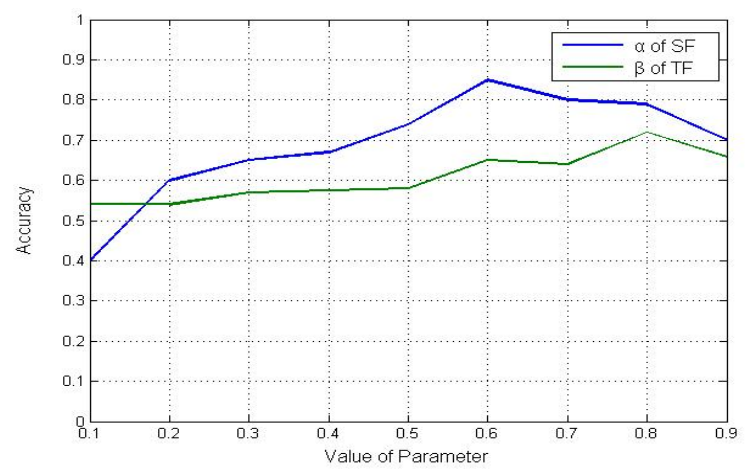

Fig. 4. Performance under different parameter.

definition of emotion influence in this paper from existing works. To quantify emotion influence, we formally define the aforementioned three factors as follows.

Structural Factor. As discussed, the propagation of information in online social network forms a tree structure, where the post publisher corresponds to the tree root, and other users who repost the message form a set of nodes including internal nodes and leaf nodes. Each internal node could form a unique subtree rooted from itself, which could be considered as an intuitive reflection of its structural influence. To formulate this factor, we consider both the relative and absolute size of subtrees. Additionally, we take the depth of subtrees into account to evaluate the density of a tree. A denser tree might indicate a higher influence. We use an exponential function to express this effect, and formally define the structural factor $S F_{u}(w)$ as follows:

$$
S F_{u}(w)=\alpha \frac{S_{u}(w)}{S(w)}+(1-\alpha) \exp \left\{-\frac{D P_{u}(w)}{\log S_{u}(w)}\right\}
$$

where $w$ denotes the original post; $S(w)$ is the size of corresponding tree; and $S_{u}(w)$ and $D P_{u}(w)$ represent the size and depth of the subtree rooted from user $u$, respectively. To determine $\alpha$, we apply decision tree to classify the influence of users, i.e., leader and follower. According to result showed in Figure 4, $\alpha$ is set to 0.6.

Temporal Factor. A user's activeness in online social networks is an important factor to reflect his/her online influence, as active users would pay more attention to online information and tend to get involved voluntarily. Moreover, based on the tree structure, the living period of online information differs from each other, where one of the influence factors is whether influential users have participated in the process of propagation. We use the time delay of a user's participation from the time of original post to measure user activeness and use the relative time ratio to describe the influence from temporal perspective. The temporal factor $T F_{u}(w)$ is introduced as follows:

$$
T F_{u}(w)=\beta \frac{L P_{u}(w)}{L P(w)}+(1-\beta) \exp \left\{-\left(t_{u}-t_{w}\right)\right\}
$$

where $L P_{u}(w)$ represents the lifespan of the interaction between user $u$ and one online post $w$, which starts from time $t_{u}$ when user $u$ makes a post $R_{w}$ that is referred to the original post $w$, and ends at time $t_{w}$ when the last repost behavior on $R_{w}$ has occurred. And $L P_{w}$ is the life cycle of the original information $w, t_{w}$ represents the starting time of information $w$ when it was first posted. The exponential function $\exp \left\{-\left(t_{u}-t_{w}\right)\right\}$ describes the time delay from the publishing of $w$ to the 
repost action of $u$. In addition, we apply the same method to determine $\beta$, and the result showed in Figure 4 denotes 0.8 as best parameter.

Emotion Change Factor. Emotion change factor is used to measure emotion change before and after a user's participation. We use $B_{u}(w, e)$ to denote the emotion distribution of post $w$ before the participation of user $u$ to repost that post, and $A_{u}(w, e)$ to denote the distribution after $u$ 's participation. Specifically, these two measurements $\left(B_{u}(w, e)\right.$ and $\left.A_{u}(w, e)\right)$ are both represented by a triple, which includes three elements and each of them indicating the ratio of positive, negative, and objective emotion respectively. The Euclidean distance is used to measure the difference between above two distributions, which is normalized by exponential function. The emotion change factor $E F_{u}(w)$ is introduced as follows:

$$
E F_{u}(w)=-\exp \left\{\sum_{e \in M}\left|B_{u}(w, e)-A_{u}(w, e)\right|\right\}+1 .
$$

Using above three factors, we can compute the emotion influence of a user systematically. It is worth noting that there are a large number of posts that have never been reposted. Therefore, it's unnecessary to compute the influence of such posts. What really attract us is the number of posts that have been reposted (denoted as $H R_{u}$ ) and the number of posts that have never been reposted (denoted as $N R_{u}$ ), because the ratio of $H R_{u}$ could denotes users' sustained influence.

\subsection{Emotion Feature Fusion}

In the case of emotion orientation, we use $E R_{u}(w)$ and $H P_{u}(e)$ to represent the relation between user $u$ and post $w$ and the preference of $u$ to emotion $e$, respectively. To characterize one's emotion orientation from the personal point of view, we need to consider these factors in an integrated manner. In other words, we use two features to classify users into three categories (i.e., positive, neutral or negative) based on machine learning method. Specifically, we use $E R_{u}$ and $H P_{u}$ to represent one's emotion orientation and introduce them as follows:

$$
\begin{gathered}
E R_{u}=\frac{\sum_{w \in W_{u}} E R_{u}(w)}{H R_{u}+N R_{u}} \\
H P_{u}=\frac{H P_{u}(P)-H P_{u}(N)}{\sum_{e \in\{P, O, N\}} H P_{u}(e)}
\end{gathered}
$$

where $H R_{u}$ and $N R_{u}$ are the numbers of user $u$ 's posts that have and have not been reposted by others respectively. Hence, the sum of $H R_{u}$ and $N R_{u}$ indicates the total posts that belongs to user $u$. Accordingly, in equation 6, we could deduce that $E R_{u}$ is the mean of one's degree of emotional characteristics. Besides, in equation 7, we attempt to measure the user's overall preference on the three emotion feelings. Specifically, if a user is more likely to post/repost positive messages, the corresponding $H P_{u}(P)$ will be larger than $H P_{u}(N)$, thus the value of $H P_{u}$ can be a positive value. As such, we can use the results of $H P_{u}$ directly as the indicators showing the user's overall emotion preference. For instance, a positive/negative value of $H P_{u}$ indicates that the user prefers positive/negative posts. If the value is close to 0 , it indicates that the user keeps a balanced emotion preference to positive/negative feeling, which also means that the user is more likely to post/repost neutral messages.

As for computing $E R_{u}$, we noticed that among all posts the user has participated, there are always a small amount of records which have extreme $E R_{u}$ values comparing to averaged $E R_{u}$ values. We regard these records as "abnormal behavior" since their corresponding emotion character values seem to be abnormal than most of the records. One example of such situation is shown in Figure 5, where each vertical line indicates the value of historical degree of emotional characteristics with a 


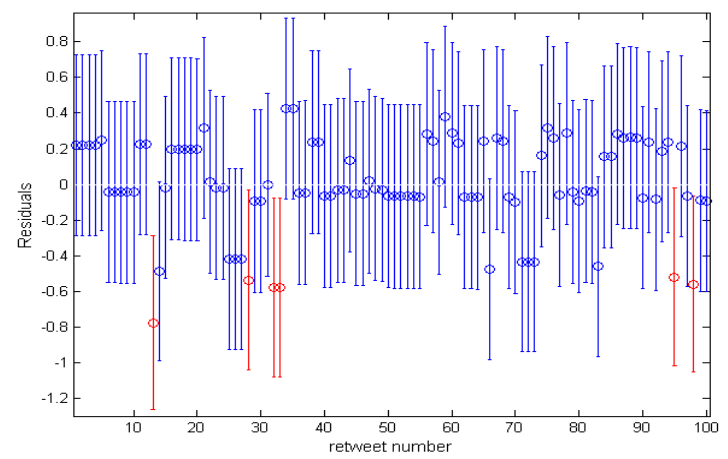

Fig. 5. Regression result of a user.

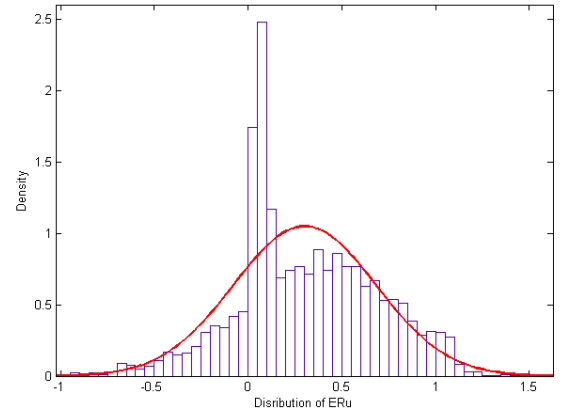

(a) Distribution of degree of emotional characteristics.

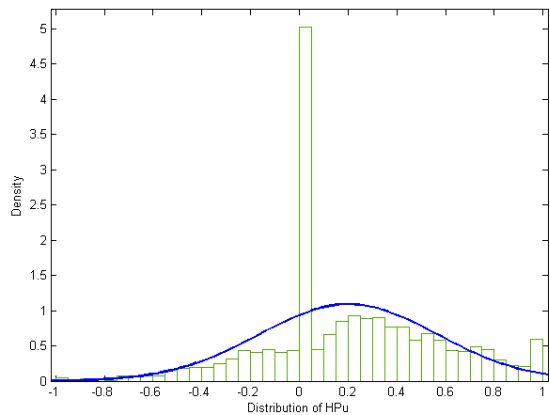

(b) Distribution of emotion preference.

Fig. 6. Distribution of orientation features.

0.95 confidence interval, and the blue lines are normal records while the red ones are considered as noises. However, $E R_{u}$ is a mean value based feature that is sensitive to outliers, and can be significantly influenced by these outliers in the dataset. More importantly, abnormal/extreme behaviors reflected by these outlier data points, no matter they are produced by accident or on purpose, do not reflect the real characteristics of users. Nevertheless, such outlier data can significantly affect our judgments since such abnormal behaviors are much more different from a user's normal behavior. In order to have a more representative value for $E R_{u}$, we need to eliminate such abnormal behaviors (outliers). To do this, we proposed a denoising process based on linear regression of least square method and set the confidence interval as 0.95 to eliminate all the outliers, which can help us prevent this feature from being biased. After denoising has completed, we plotted the distribution of the two features in Figure 6, which includes the distribution of degree of emotional characteristics in Figure 6(a) and the user's accumulated emotion preference distribution in Figure 6(b) respectively. In order to give a more visualized tendency of the data distribution, we draw fitting curves using Gaussian formulation, from which we could observe that the majority of users are inclined to be neutral.

As different factors have different impacts on the user's emotion influence, we need to evaluate the weight of each factor before fusing them together. Based on the tree structure of a post, we 
first obtain the distribution of each factor, and then combine them linearly. We introduce another metric, named as ratio of reposted, to represent the portion of a user's messages (including both original posts and reposts) that has been reposted by others. This metric is used to adjust the effect of individual abnormal reposts. The integrated emotion influence is calculated as follows, where $\phi_{1}, \phi_{2}$, and $\phi_{3}$ represent the weight of the structural factor, the temporal factor and the emotion change factor respectively:

$$
E I_{u}=\frac{\sum_{w \in W_{u}} \phi_{1} S F_{u}(w)+\phi_{2} T F_{u}(w)+\phi_{3} E F_{u}(w)}{H R_{u}}\left(1+\frac{H R_{u}}{H R_{u}+N R_{u}}\right)
$$

according to the above definition, we could deduce that $E I_{u}$ contains two parts. Firstly, we calculate the weighted average value of the intensity of emotion influence by summing the proposed three features, and then divided the sum by the number of all reposts $\left(H R_{u}\right)$ that belongs to user $u$ 's post $w$. Secondly, we introduce the repost rate $\left(\frac{H R_{u}}{H R_{u}+N R_{u}}\right)$ to denote the ability such influence could affect others. A higher value of the rate indicates a higher ability one's post could affect others. And finally, we multiply these two values to obtain a comprehensive description of user's emotion influence.

\subsection{Emotion Role Detection Model}

To classify a user into one of the six different emotion roles, we propose a Multi-dimensional Emotion Role Mining model (MERM), which integrates both emotion orientation and emotion influence to identify one's emotion role.

In the case of emotion influence, there is no standard evaluation method. When analyzing social networks, centrality is a widely used metric to measure user's influence including degree centrality, closeness centrality, and betweenness centrality, where the threshold selection has been used to identify users with different influence. Inspired by such scheme, to get the contribution weight of each feature, we first represent all the results computed by equation 3, 4, 5 as vectors:

$$
\begin{gathered}
S F=\left(\begin{array}{llll}
S F\left(w_{1}\right), & S F\left(w_{2}\right), & \cdots, & S F\left(w_{n}\right)
\end{array}\right) \\
T F=\left(\begin{array}{llll}
T F\left(w_{1}\right), & T F\left(w_{2}\right), & \cdots, & T F\left(w_{n}\right)
\end{array}\right) \\
E F=\left(\begin{array}{llll}
E F\left(w_{1}\right), & E F\left(w_{2}\right), & \cdots, & E F\left(w_{n}\right)
\end{array}\right)
\end{gathered}
$$

To evaluate the weight of each feature, we explore the global statistics of their distributions. The standard deviation measures the dispersion of target data, where a smaller value indicates stronger aggregation degree and poor discrimination degree. Therefore, the feature with relatively larger standard deviation is more important. However, the discrimination ability of standard deviation is based on the assumption that the target data has the same dimension. To solve this problem, we introduce the Coefficient of Variance (CV), to denote the division of standard deviation over mean. This statistic parameter can eliminate the effect caused by different dimensions and provide a consistent and comparable measurement. By applying the Coefficient of Variance (CV) algorithm, the values of each $\phi_{i}, i \in 1,2,3$ in equation 8 are deduced. As a result, we can get the emotion influence for each user. According to previous work [26], we set $\mu+2 \sigma$ as the threshold to identify emotion leaders and followers. The users whose $E I_{u}$ is greater than the threshold are recognized as emotion leaders while others as followers.

We build MERM based on decision tree model, which is suitable for high quality classification under less features. In our study, we use the user set we constructed as root sample, and each user is characterized by three features, namely $E R_{u}, H P_{u}$, and $E I_{u}$ respectively. We select the mainstream algorithm C4.5 for emotion role identification. It splits target data into subcategories based on 
normalized information gain, and applies J48, an open source Java implementation of C4.5 in WEKA [11]. For each user we first calculate their influence as $E I_{u}^{\prime}$.

$$
E I_{u}^{\prime}= \begin{cases}1 & E I_{u} \geq \mu+2 \sigma \\ 0 & E I_{u}<\mu+2 \sigma\end{cases}
$$

Then we build a vector $U_{u}=\left(E R_{u}, H P_{u}, E I_{u}^{\prime}\right)$ as the input to building decision tree. Here $U_{u}^{j}$ is the $j$ th feature, and the entropy and information gain formed the basis of the method. The formal notations are introduced as follows:

$$
\begin{gathered}
\operatorname{Entropy}(U)=\sum_{j=1}^{3}-U^{j} \log _{2} U^{j} \\
\operatorname{Gain}(U, A)=\operatorname{Entropy}(U)-\sum_{v \in V(A)} \frac{\left|U_{v}\right|}{U} \operatorname{Entropy}\left(U_{v}\right)
\end{gathered}
$$

where $U$ and $A$ represent instance set of users, attribute of vectors, respectively. $U_{v}$ denotes the user set whose attribute value of $A$ equals $v$. In C4.5, the ratio of information gains is used to choose attributes, which is calculated as follows:

$$
\operatorname{GainRatio}(U, A)=\frac{\operatorname{Gain}(U, A)}{\operatorname{Entropy}(U, A)}
$$

To create a node N, we label the node as attribute with the highest GainRatio, and perform this procedure recursively to build a decision tree. Finally, we use this tree to classify the users into six different categories, i.e., six emotion roles.

\section{EXPERIMENTAL RESULTS}

In this section, we conduct detailed analysis on our proposed features and methods. We first provide the overview of the dataset, and then we describe the evaluations on our proposed features. Following that, we conduct experiments on detecting user's emotion orientation and measuring user's emotion influence respectively. Finally, we apply all the features to classify a user's emotion role into six categories to demonstrate the effectiveness of our model.

Before demonstrating any experimental results, we first illustrate the key procedure for model training and parameter tuning in our experiments. Specifically, the dataset is initially divided into training and testing datasets before any further experiments are conducted. Based on training dataset, we simultaneously train the model and tune the parameters through the following procedures. Given one possible parameter setting, 1) we first calculate the features for each instance in the training dataset, and 2) we then apply decision tree algorithm using these instance in a 10-fold cross validation manner, notice that all the parameters are now determined/unchanged and we are using training dataset lonely, to see the classification accuracy under such parameter setting. And we repeat the above two steps by changing the parameters' values to determine the best parameter setting when the model reaches its highest classification accuracy. Since the parameters are unchanged during the validation process, thus we do not select parameters in each round of the validation. In fact, all the parameters we proposed only influence how we transfer the raw dataset into feature-based instances for training purpose, and we do not tune the model's inner parameters. Detailed experimental results can be found in the following sections. 
Table 1. Components of XML structure.

\begin{tabular}{c|c}
\hline Property & Description \\
\hline org_id & ID of original post \\
\hline org_time & Release time of original post \\
\hline org_emotion & Emotion of original post \\
\hline w_id & ID of current repost \\
\hline w_time & Release time of current repost \\
\hline w_text & Textual content of current post \\
\hline w_emotion & Emotion of current repost \\
\hline u_id & ID of the user \\
\hline p_name & Parent name of current repost \\
\hline
\end{tabular}

\subsection{Date Set}

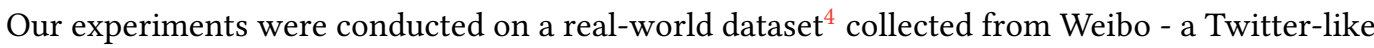
micro-blog service in China. We used APIs provided by Weibo service to crawl repost information within a specified time interval (from Jan. 2014 to June.2014). To make the data set manageable, we first extract all original posts in our data set to form a list of roots of repost trees, and then classify reposts into different levels based on the number of identifiers (i.e., "//@”) in the repost text. After this we are able to construct the tree structure for each Weibo post. The process is presented as follows:

(1) Add the 1st-level reposts onto original posts directly based on the original-post information within each raw record derived through APIs.

(2) Add the next level reposts onto its parent nodes based on both the original-post information and the parent user name following identifier “//@”. Specifically, the original-post information is used to find which tree the current repost belongs to, and the parent user name is used to locate the direct parent of the current repost.

(3) Recursively repeat Step 2 until all the high level reposts are added onto their parent nodes.

Based on the above procedure, we obtain a list of repost trees and save them as XML files. Table 1 presents the properties and descriptions used to construct each repost. From this data set we extract the same nodes (a node is actually a user) to build each individual's history profile. Those nodes (i.e., users) that appeared in at least 5 repost trees are selected as the experimental entity. Figure 7 shows an example of user profile. To generate the ground truth for emotion role classification we have manually labeled the users as one of the six emotion roles according to their comments and repost trees. The emotion role labeling and three principles are described below:

(1) Users' repost profile and statistical results (i.e., distribution of three different emotions) of repost tree related to each repost are provided as labeling evidence.

(2) Volunteers label users as one of six emotion roles according to their historical behaviors including comments, static emotions and statistical results.

(3) Each user is reviewed by three different volunteers to eliminate personal bias, and the majority opinion will be selected as the final labeling result. If there exists a conflict in determining the label, all the volunteers are required to have a discussion to reach an agreed conclusion.

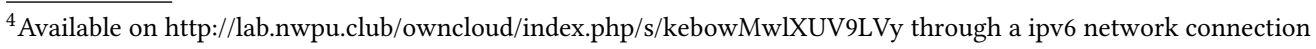




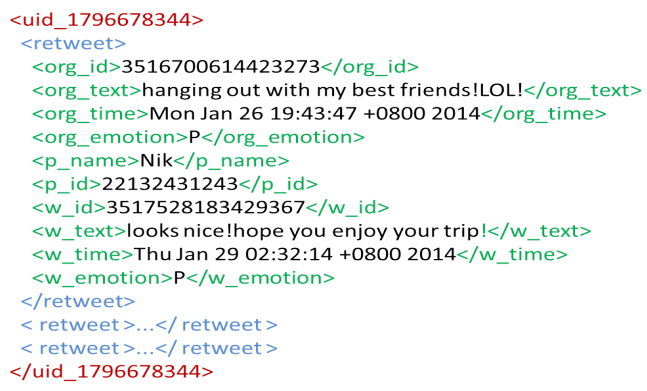

Fig. 7. An example of user profile.

Table 2. Statistics of the Weibo data set.

\begin{tabular}{c|c}
\hline Property & Quantity \\
\hline Number of trees & 7,096 \\
\hline Number of users & 19,389 \\
\hline Number of users labeled as positive & 4,573 \\
\hline Number of users labeled as neutral & 12,367 \\
\hline Number of users labeled as negative & 2,448 \\
\hline Number of users labeled as leader & 131 \\
\hline Number of users labeled as follower & 19,258 \\
\hline Number of total reposts & $2,606,970$ \\
\hline Number of reposts from selected users & 175,902 \\
\hline
\end{tabular}

To keep the consistency of the labeling procedure from different volunteers, we apply Cohen's kappa coefficient ${ }^{5}$ to calculate their pair-wise inter-agreement value $(I A)$ as follows:

$$
I A\left(V_{i}, V_{j}\right)=\frac{P_{o}-P_{e}}{1-P_{e}}
$$

where $P_{o}$ is the relative observed agreement among volunteer $V_{i}$ and $V_{j}$ and $P_{e}$ is the hypothetical probability of chance agreement. Detailed calculation method can refer to [18]. According to [10], a larger Kappa coefficient ( $I A$ in our work) indicates a higher reliability the labeling results are. And to obtain high reliable results, we apply 0.8 as the threshold for $I A$ w.r.t the detailed discussion in [10]. Based on the above principles, we recruited 16 volunteers (master students majored in computer science) to conduct data labeling. Before labeling all the users, the volunteers are asked to label a sample group of users to determine their pair-wise inter-agreement values, and if any volunteersâĂŹ pair-wise inter-agreement value is lower than threshold (0.8), the volunteers are re-trained until all the pair-wise inter-agreement is lager than threshold (0.8). Only then can we proceed to label the whole dataset in a more reliable manner. In specific, the volunteers first judged whether a user is a leader or a follower, and then decided the user's emotion preference (positive, negative, or objective). In the first step, 18,807 users were labeled with same emotion roles. As for deciding a user's emotion preference, 17,256 users were given three same emotion characters by volunteers, 1,648 users were labeled with two different emotion characters, i.e., 2 same and 1 different, and 485 users were given three distinct labels by volunteers. For those users with three

\footnotetext{
${ }^{5}$ https://en.wikipedia.org/wiki/Cohen\%27s_kappa
} 


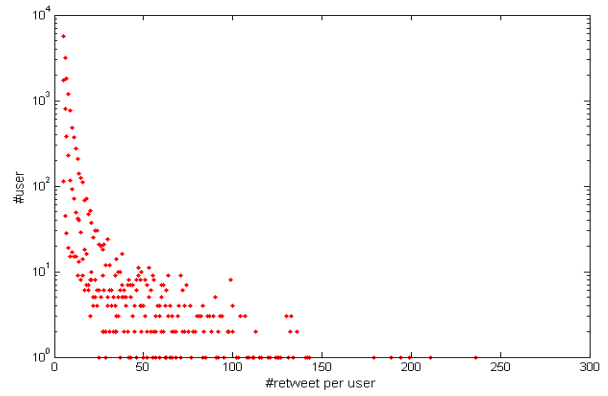

Fig. 8. Distribution of the number of reposts.

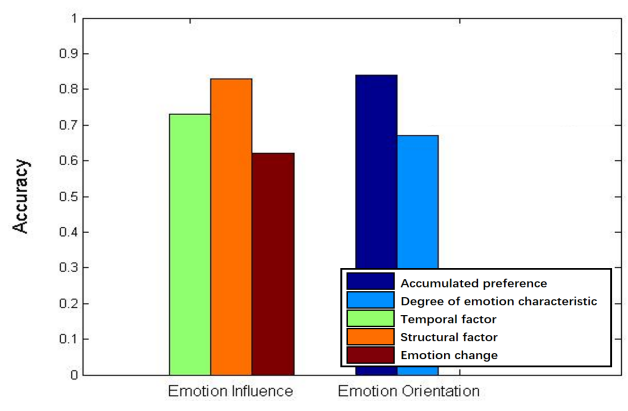

Fig. 9. Classification performance of each feature.

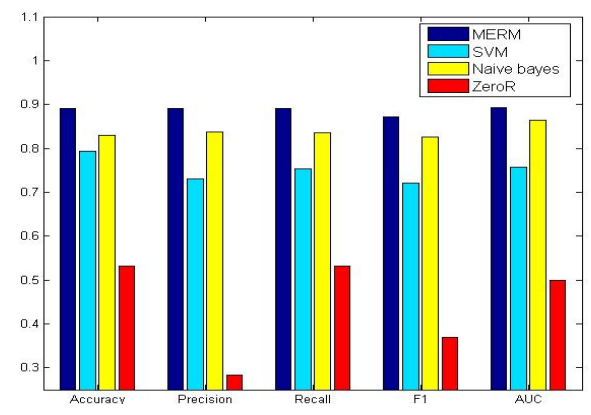

Fig. 10. Performance comparison of four algorithms.

different labels, all the corresponding volunteers were required to have an extra discussion to reach an agreed conclusion on the user's label. Some related statistics result of data set are shown in Table 2. To explore the characteristics of users' repost behaviors, we take a statistical analysis about the distribution of repost number as shown in Figure 8, which follows the power-law distribution well and indicating that a small part of users got involved in most of the reposts. The average repost number is 9.07 .

\subsection{Feature Evaluation}

In our MERM model we consider both emotion orientation and emotion influence from two and three aspects respectively. To evaluate their contribution to emotion role classification, we adopted decision tree to predict attendance using each category of proposed features separately and selected the accuracy as performance measure. The results are shown in Figure 9. The results show that different features have distinct performances. For emotion orientation classification, the historical preference achieved the best performance with an accuracy of 0.84 , while the degree of emotional characteristics achieved 0.67 . For emotion influence, the structural factor, temporal factor and emotion change factor achieved the accuracy of $0.83,0.73$ and 0.62 respectively.

\subsection{Emotion Orientation Evaluation}

\subsubsection{Emotion Orientation Identification.}

To identify emotion roles with different emotion orientations, we apply decision-tree-based MERM method and three other different classification algorithms including SVM, Naïve Bayes, and ZeroR to classify users into three categories, i.e., positive, neutral and negative. 10 -fold cross validation is 


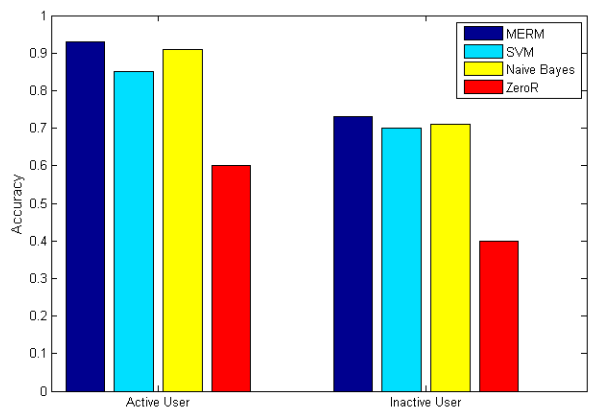

Fig. 11. Performance for two types of users.

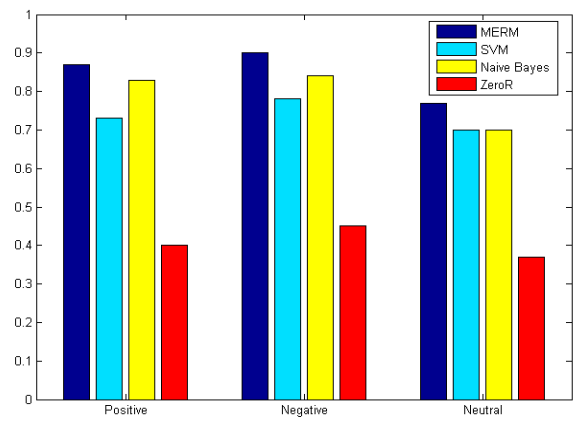

Fig. 12. Performance for three emotion orientations.

adopted to perform classification. All these tests were performed based on WEKA [11], and the results are shown in Figure 10. We compared the performance of the selected algorithms based on different measures, including accuracy, precision, recall, F-value, and AUC. As can be seen in Figure 10, the MERM achieves the best performance for all measures. In particular, the accuracy of MERM is up to 0.891 . The accuracy of Naïve Bayes is 0.835 . SVM has the third highest accuracy of 0.753 , and ZeroR takes the last position.

\subsubsection{Performance Evaluation for Different Users.}

We noticed that the activeness of online social network users is quite different from each other. Some of them use online services much more frequently than the others. To evaluate the performance of the developed identification method, we conduct classification experiments based on two types of users, i.e., active users and inactive users. In our data set, as the average repost number of each user is 9.07, we select users who made at least 9 reposts per month as active users while the others as inactive users. The experimental result, as shown in Figure 11, shows that the overall performance of active users is much better than that of inactive users. Specifically, in the case of active users, the accuracy of MERM is up to 0.93 . There might be two reasons why the accuracy of active users outperforms that of inactive users. The first one is that active users tend to interact more with online information and are used to express their emotions, i.e., their online behaviors are more regular compared with that of inactive users. The second one is that we do not have sufficient evidence for inactive users when identifying their emotion orientations.

\subsubsection{Performance Evaluation for Different Orientation.}

To evaluate the proposed method, we design a set of experiments to identify three different orientations. The result, as shown in Figure 12, reveals intuitively that the accuracy of neutral orientation is lower than the other two orientations. Specifically, in the case of positive orientation, the accuracy of MERM, Naïve Bayes, and SVM are 0.87, 0.83, and 0.73, respectively. In the case of negative orientations, MERM again achieves a highest accuracy of 0.91 , while the accuracy of Naïve Bayes and SVM are 0.84 and 0.78 . However, for neutral orientation, the highest accuracy of decision tree is only 0.77 . Many reasons may cause such an imbalanced classification result. In this paper, we consider the following possible reason that lead to this result. The features used in our work are not so significant in representing neutral orientation as those used in positive or negative orientations, since positive and negative are all extreme feeling while neutral is not. Table 3 presents all results. 
Table 3. Result of performance evaluation.

\begin{tabular}{c|c|c|c|c}
\hline & MERM & SVM & Naïve bayes & ZeroR \\
\hline Active user & 0.93 & 0.80 & 0.83 & 0.61 \\
\hline Inactive user & 0.79 & 0.68 & 0.71 & 0.43 \\
\hline Positive & 0.87 & 0.73 & 0.83 & 0.40 \\
\hline Negative & 0.91 & 0.78 & 0.84 & 0.45 \\
\hline Neutral & 0.77 & 0.70 & 0.71 & 0.37 \\
\hline
\end{tabular}

\subsection{Emotion Influence Evaluation}

We compute each factor using the developed calculation equations and conduct statistical analysis about their distribution. The result, as shown in Figure 13, indicates that three factors have different characteristics. Figure 13(a) shows a Gaussian like distribution. Specifically, the value of structural factor gathers around 0.3 to 0.4 , and its mean value and standard deviation are 0.331 and 0.058 . Similarly, we also use Gaussian distribution to fit the trend of the temporal factor, as in Figure 13(b). The mean value is 0.140 and the standard deviation is 0.142 . For emotion factor in Figure 13(c), the curve goes upward firstly and then falls down rapidly. The reason might be that, compared with the whole repost tree, the sizes of most subtrees related to users are quite small. In fact, subtrees with only couples of nodes account for a large portion in our data set - a common phenomenon in online social networks. As such, the emotions change before and after a specific user's participation is inclined to be obvious. Nevertheless, this is not representative because the limited number of nodes within these small subtrees is not able to represent opinions of a relatively large scale. The downward trend at the right part in Figure 13(c) reflects the effect of total reversal of emotions. This is a hypothesized ideal state but hardly happen in the real-world scenarios. We use generalized extreme value distribution to fit the trend, and the mean value and standard deviation are 0.637 and 0.252 .

Based on the above feature calculation and analysis, the weight of each feature is computed as coefficient of variance as follows: $\phi_{1}=0.175, \phi_{2}=1.014, \phi_{3}=0.396$. We then compute emotion influence. The result is shown in Figure 14. We use $\mu+2 \sigma$ according to [47] to set the threshold to identify emotion leaders and followers, which is 1.41 in this study. Among 19,389 users in our dataset we identified 105 users as emotion leaders and the others as emotion followers. The classification accuracy of emotion leaders and followers are 0.87 and 0.93 , respectively. One possible reason for

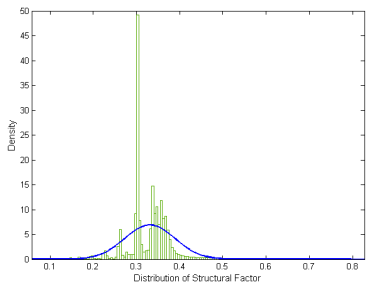

(a) Distribution of structural factor

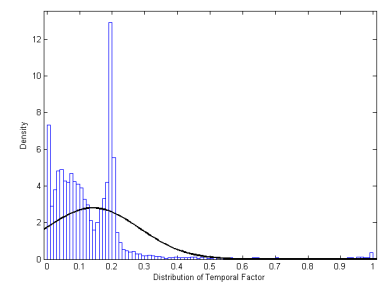

(b) Distribution of temporal factor

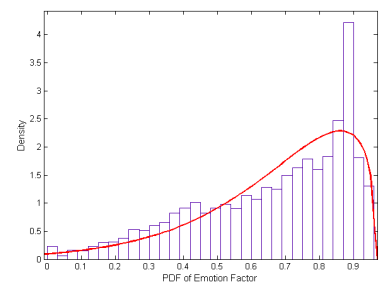

(c) Distribution of emotion change factor

Fig. 13. Analysis of emotion influence factors. 


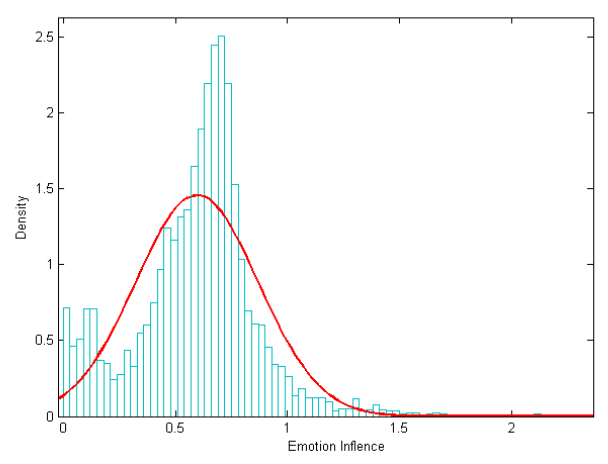

Fig. 14. Distribution of emotion influence.

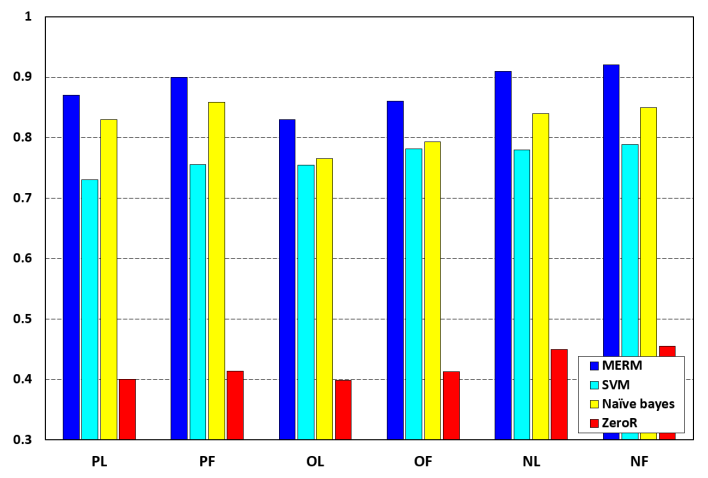

Fig. 15. Accuracy for detecting six emotion roles.

this imbalanced accuracy is that, there are totally 19,258 labeled followers while that is only 131 for labeled leaders, thus it could have much more significant evidences for our method to extract effective features to model followers than leaders.

\subsection{Emotion Role Evaluation}

Our previous evaluations proved that the proposed features are all effective and useful. And by integrating all these features, we are able to classify users into six fine-grained emotion roles. The result is showed in Figure 15, where PL is Positive Leader, PF is Positive Follower, OL is Neutral Leader, OF is Neutral Follower, NL is Negative Leader, and NF is Negative Follower. Overall, our proposed model MERM outperforms other models in all classes. In details, the result of MERM shows that the accuracy of negative roles, i.e. 0.91 and 0.92 for leaders and followers respectively, is higher than other emotion roles. The accuracy of neutral follower is 0.86 which is 0.03 higher than neutral leader. For positive emotion, both accuracy of leaders and followers are in the middle of others, which achieved 0.87 and 0.90 . The average accuracy of classification is up to 0.91 . Except our model, Naive Bayes performs the best, followed by SVM, and ZeroR performs the worst. The experimental results across all these models in classifying different emotion roles demonstrated the effectiveness of our method.

\section{DISCUSSION}

In summary, according to the above experiments, our proposed features and methods are effective in detecting user's emotion roles. Specifically, 1) our method has achieved 0.891 accuracy in classifying user's emotion orientation into three categories: positive, negative, and objective respectively; 2) we have obtained 0.87 and 0.93 accuracy in recognizing whether a user is leader or follower; 3) by applying the integrated features and the proposed MERM model, we have achieved 0.91 averaged accuracy in detecting the user's emotion role, which includes six types: Positive Leader/Follower (PL/PF), Negative Leader/Follower (NL/NF), and Objective Leader/Follower (OL/OF). All these results generated by our proposed MERM model outperform the other models including SVM, Naive Bayes, and the baseline method ZeroR. As our model is the extension from decision tree-based model, it inherits the benefits from typical decision tree model, such that our model is able to implicitly perform feature selection process while other models can not, which can be of great importance to improve the performance. In addition, we have enhanced our decision tree-based model to support feature fusion process and parameter tunning procedure. 
However, there are also a few issues that could be discussed. Firstly, our dataset is still relatively small for deeper analysis. We could collect more users not only from Weibo, but also from other social network platforms like Twitter and Facebook, thus we are able to conduct more comprehensive analysis on the experimental results, for example, to discover the reasons why imbalanced performance appears. Secondly, some parameters in our study are determined from experimental tuning, we need to propose some learning formulas to learn such parameters to come up with more convincing results. Finally, some other property of a user could be taken into account, for instance, the use of tree-based structure of posts and reposts, we need to discover more features to make the model more robust.

\section{CONCLUSIONS AND FUTURE WORK}

In this paper, we addressed the emotion role detection problem - a new area of online social network analysis. To this end we have defined the concept of emotion role from two different perspectives, namely emotion orientation and emotion influence. We developed and quantified the emotion role metrics by introducing five different factors. Built upon these analyses we further developed the MERM model to identify emotion roles. Our work is the very first attempt to explore emotion roles in online social networks and shed a light on the analysis of online user behaviors. It also provides significant insights which may contribute to personalized recommendation and public opinion detection.

In the future, we will introduce more features into our model, such as friend relationships, to improve the recognition performance. Furthermore, we plan to apply some related methods, such as under-sampling [39], to deal with the imbalanced dataset problem in our future work. To further explore the usefulness of our proposed model, we will investigate the application of our work to other online communities, such as Reddit, Quora, and Digg, to discover emotion leaders and followers. It is also our intention to develop a system like [5] based on our model, which could suggest a list of users for accomplishing a specific task, such as maximizing the message's positive propagation in the online social network.

\section{ACKNOWLEDGMENTS}

This work was supported in part by the National Basic Research Program of China (No. 2015CB352400), the National Science Fund for Distinguished Young Scholars (No. 61725205), and the National Natural Science Foundation of China (No. 61332005, 61772428).

\section{REFERENCES}

[1] Afra Abnar, Mansoureh Takaffoli, Reihaneh Rabbany, and Osmar R Zaïane. 2015. SSRM: structural social role mining for dynamic social networks. Social Network Analysis and Mining 5, 1 (2015), 1-18.

[2] Haji Binali and Vidyasagar Potdar. 2012. Emotion detection state of the art. In Proceedings of the CUBE International Information Technology Conference. ACM, 501-507.

[3] Freimut Bodendorf and Carolin Kaiser. 2009. Detecting opinion leaders and trends in online social networks. In Proceedings of the 2nd ACM workshop on Social web search and mining. ACM, 65-68.

[4] Felipe Bravo-Marquez, Marcelo Mendoza, and Barbara Poblete. 2013. Combining strengths, emotions and polarities for boosting Twitter sentiment analysis. In Proceedings of the Second International Workshop on Issues of Sentiment Discovery and Opinion Mining. ACM, 2.

[5] Huihui Chen, Bin Guo, Zhiwen Yu, and Liming Chen. 2015. CrowdPic: a multi-coverage picture collection framework for mobile crowd photographing. In Ubiquitous Intelligence and Computing and 2015 IEEE 12th Intl Conf on Autonomic and Trusted Computing and 2015 IEEE 15th Intl Conf on Scalable Computing and Communications and Its Associated Workshops (UIC-ATC-ScalCom), 2015 IEEE 12th Intl Conf on. IEEE, 68-76.

[6] Huihui Chen, Bin Guo, Zhiwen Yu, Liming Chen, and Xiaojuan Ma. 2017. A generic framework for constraint-driven data selection in mobile crowd photographing. IEEE Internet of Things fournal 4, 1 (2017), 284-296. 
[7] Anqi Cui, Haochen Zhang, Yiqun Liu, Min Zhang, and Shaoping Ma. 2013. Lexicon-based sentiment analysis on topical chinese microblog messages. In Semantic Web and Web Science. Springer, 333-344.

[8] Kurt Junshean P Espinosa and Alma Mae J Bernales. 2014. Characterizing influence factors affecting emotion diffusion in facebook. In Proceedings of the World Congress on Engineering and Computer Science, Vol. 2.

[9] Noah E Friedkin and Eugene C Johnsen. 1999. Social influence networks and opinion change. Advances in group processes 16, 1 (1999), 1-29.

[10] KL Gwet. 2012. Benchmarking inter-rater reliability coefficients. Handbook of inter-Rater reliability: the definitive guide to measuring the extent of agreement among raters. 3rd ed. Gaithersburg, MD: Advanced Analytics 200 (2012), 164-180.

[11] Mark Hall, Eibe Frank, Geoffrey Holmes, Bernhard Pfahringer, Peter Reutemann, and Ian H Witten. 2009. The WEKA data mining software: an update. ACM SIGKDD explorations newsletter 11, 1 (2009), 10-18.

[12] Xia Hu, Jiliang Tang, Huiji Gao, and Huan Liu. 2013. Unsupervised sentiment analysis with emotional signals. In Proceedings of the 22nd international conference on World Wide Web. ACM, 607-618.

[13] Xia Hu, Lei Tang, Jiliang Tang, and Huan Liu. 2013. Exploiting social relations for sentiment analysis in microblogging. In Proceedings of the sixth ACM international conference on Web search and data mining. ACM, 537-546.

[14] Anup K Kalia, Norbou Buchler, Diane Ungvarsky, Ramesh Govindan, and Munindar P Singh. 2014. Determining team hierarchy from broadcast communications. In International Conference on Social Informatics. Springer, 493-507.

[15] Suin Kim, JinYeong Bak, and Alice Oh. 2012. Discovering emotion influence patterns in online social network conversations. SIGWEB Newsl.,(Autumn) 3 (2012), 1-3.

[16] Fang-Fei Kuo, Meng-Fen Chiang, Man-Kwan Shan, and Suh-Yin Lee. 2005. Emotion-based music recommendation by association discovery from film music. In Proceedings of the 13th annual ACM international conference on Multimedia. ACM, 507-510.

[17] Lu Lin, Jianxin Li, Richong Zhang, Weiren Yu, and Chenggen Sun. 2014. Opinion Mining and Sentiment Analysis in Social Networks: A Retweeting Structure-Aware Approach. In Utility and Cloud Computing (UCC), 2014 IEEE/ACM 7th International Conference on. IEEE, 890-895.

[18] Mary L McHugh. 2012. Interrater reliability: the kappa statistic. Biochemia medica 22, 3 (2012), 276-282.

[19] Qiaozhu Mei, Xu Ling, Matthew Wondra, Hang Su, and ChengXiang Zhai. 2007. Topic sentiment mixture: modeling facets and opinions in weblogs. In Proceedings of the 16th international conference on World Wide Web. ACM, 171-180.

[20] Andrius Mudinas, Dell Zhang, and Mark Levene. 2012. Combining lexicon and learning based approaches for conceptlevel sentiment analysis. In Proceedings of the first international workshop on issues of sentiment discovery and opinion mining. ACM, 5.

[21] Subhabrata Mukherjee, Gaurab Basu, and Sachindra Joshi. 2013. Incorporating author preference in sentiment rating prediction of reviews. In Proceedings of the 22nd International Conference on World Wide Web. ACM, 47-48.

[22] Bo Pang and Lillian Lee. 2004. A sentimental education: Sentiment analysis using subjectivity summarization based on minimum cuts. In Proceedings of the 42nd annual meeting on Association for Computational Linguistics. Association for Computational Linguistics, 271.

[23] Bo Pang, Lillian Lee, and Shivakumar Vaithyanathan. 2002. Thumbs up?: sentiment classification using machine learning techniques. In Proceedings of the ACL-02 conference on Empirical methods in natural language processing-Volume 10. Association for Computational Linguistics, 79-86.

[24] Darren Quinn, Liming Chen, and Maurice Mulvenna. 2012. Social Network Analysis-A Survey. International fournal of Ambient Computing and Intelligence 4, 3 (2012), 46-58.

[25] Farag Saad. 2014. Baseline evaluation: an empirical study of the performance of machine learning algorithms in short snippet sentiment analysis. In Proceedings of the 14th International Conference on Knowledge Technologies and Data-driven Business. ACM, 6.

[26] M Zubair Shafiq, Muhammad U Ilyas, Alex X Liu, and Hayder Radha. 2013. Identifying leaders and followers in online social networks. IEEE Journal on Selected Areas in Communications 31, 9 (2013), 618-628.

[27] Juan Shi, Kin Keung Lai, Ping Hu, and Gang Chen. 2017. Understanding and predicting individual retweeting behavior: Receiver perspectives. Applied Soft Computing (2017).

[28] Yoshihiko Suhara, Yinzhan Xu, and Alex'Sandy' Pentland. 2017. Deepmood: Forecasting depressed mood based on self-reported histories via recurrent neural networks. In Proceedings of the 26th International Conference on World Wide Web. International World Wide Web Conferences Steering Committee, 715-724.

[29] Abhay Sukumaran, Stephanie Vezich, Melanie McHugh, and Clifford Nass. 2011. Normative influences on thoughtful online participation. In Proceedings of the SIGCHI Conference on Human Factors in Computing Systems. ACM, 3401-3410.

[30] Chenhao Tan, Lillian Lee, Jie Tang, Long Jiang, Ming Zhou, and Ping Li. 2011. User-level sentiment analysis incorporating social networks. In Proceedings of the 17th ACM SIGKDD international conference on Knowledge discovery and data mining. ACM, 1397-1405.

[31] Duyu Tang, Bing Qin, Furu Wei, Li Dong, Ting Liu, and Ming Zhou. 2015. A joint segmentation and classification framework for sentence level sentiment classification. IEEE/ACM Transactions on Audio, Speech, and Language Processing 
23, 11 (2015), 1750-1761.

[32] Jie Tang, Yuan Zhang, Jimeng Sun, Jinhai Rao, Wenjing Yu, Yiran Chen, and AC M Fong. 2012. Quantitative study of individual emotional states in social networks. IEEE Transactions on Affective Computing 3, 2 (2012), 132-144.

[33] Simo Editha Tchokni, Diarmuid O Séaghdha, and Daniele Quercia. 2014. Emoticons and Phrases: Status Symbols in Social Media.. In ICWSM.

[34] Di Wang, Alistair Sutcliffe, and Xiao-Jun Zeng. 2011. A trust-based multi-ego social network model to investigate emotion diffusion. Social Network Analysis and Mining 1, 4 (2011), 287-299.

[35] Jingang Wang, Dandan Song, Lejian Liao, Wei Zou, Xiaoqing Yan, and Yi Su. 2013. The Chinese bag-of-opinions method for hot-topic-oriented sentiment analysis on Weibo. In Semantic Web and Web Science. Springer, 357-367.

[36] Zhitao Wang, Zhiwen Yu, Liming Chen, and Bin Guo. 2014. Sentiment detection and visualization of Chinese micro-blog. In Data Science and Advanced Analytics (DSAA), 2014 International Conference on. IEEE, 251-257.

[37] Dingqi Yang, Daqing Zhang, Zhiyong Yu, Zhiwen Yu, and Djamal Zeghlache. 2014. SESAME: mining user digital footprints for fine-grained preference-aware social media search. ACM Transactions on Internet Technology (TOIT) 14, 4 (2014), 28

[38] Yang Yang, Jia Jia, Shumei Zhang, Boya Wu, Qicong Chen, Juanzi Li, Chunxiao Xing, and Jie Tang. 2014. How do your friends on social media disclose your emotions?. In $A A A I$, Vol. 14. 1-7.

[39] Show-Jane Yen and Yue-Shi Lee. 2006. Under-sampling approaches for improving prediction of the minority class in an imbalanced dataset. Intelligent Control and Automation (2006), 731-740.

[40] Fei Yi, Zhiwen Yu, Huihui Chen, He Du, and Bin Guo. 2018. Cyber-physical-social collaborative sensing: from single space to cross-space. Frontiers of Computer Science (2018), 1-14.

[41] Zhiwen Yu, Zhitao Wang, Liming Chen, Bin Guo, and Wenjie Li. 2016. Featuring, Detecting, and Visualizing Human Sentiment in Chinese Micro-Blog. ACM Transactions on Knowledge Discovery from Data (TKDD) 10, 4 (2016), 48.

[42] Zhiwen Yu, Zhu Wang, Huilei He, Jilei Tian, Xinjiang Lu, and Bin Guo. 2015. Discovering information propagation patterns in microblogging services. ACM Transactions on Knowledge Discovery from Data (TKDD) 10, 1 (2015), 7.

[43] Zhiwen Yu, Fei Yi, Qin Lv, and Bin Guo. 2018. Identifying On-site Users for Social Events: Mobility, Content, and Social Relationship. IEEE Transactions on Mobile Computing (2018).

[44] Guangchao Yuan, Pradeep K Murukannaiah, Zhe Zhang, and Munindar P Singh. 2014. Exploiting sentiment homophily for link prediction. In Proceedings of the 8th ACM Conference on Recommender systems. ACM, 17-24.

[45] Zhongwu Zhai, Hua Xu, and Peifa Jia. 2008. Identifying opinion leaders in BBS. In Web Intelligence and Intelligent Agent Technology, 2008. WI-IAT'08. IEEE/WIC/ACM International Conference on, Vol. 3. IEEE, 398-401.

[46] Ying Zhang, Ning Zhang, Luo Si, Yanshan Lu, Qifan Wang, and Xiaojie Yuan. 2014. Cross-domain and cross-category emotion tagging for comments of online news. In Proceedings of the 37th international ACM SIGIR conference on Research \& development in information retrieval. ACM, 627-636.

[47] Yuchen Zhao, Guan Wang, Philip S Yu, Shaobo Liu, and Simon Zhang. 2013. Inferring social roles and statuses in social networks. In Proceedings of the 19th ACM SIGKDD international conference on Knowledge discovery and data mining. ACM, 695-703.

Received June 2017; revised September 2017; accepted February 2018 